NBER WORKING PAPER SERIES

\title{
BUBBLE TROUBLES? RATIONAL STORAGE, MEAN REVERSION AND RUNS IN COMMODITY PRICES.
}

\author{
Eugenio S. A. Bobenrieth \\ Juan R. A. Bobenrieth \\ Brian D. Wright \\ Working Paper 19037 \\ http://www.nber.org/papers/w19037
}

\author{
NATIONAL BUREAU OF ECONOMIC RESEARCH \\ 1050 Massachusetts Avenue \\ Cambridge, MA 02138 \\ May 2013
}

Work on this paper was funded by the Energy Biosciences Institute and by CONICYT/Fondo Nacional de Desarrollo Científico y Tecnológico (FONDECYT) Project 1090017. Eugenio Bobenrieth's research for this paper was done partially when he was a professor at Universidad de Concepción, Chile. Eugenio Bobenrieth acknowledges partial financial support from Grupo Security through Finance UC, and from Project NS 100046 of the Iniciativa Científica Milenio of the Ministerio de Economía, Fomento y Turismo, Chile. The views expressed herein are those of the authors and do not necessarily reflect the views of the National Bureau of Economic Research.

At least one co-author has disclosed a financial relationship of potential relevance for this research. Further information is available online at http://www.nber.org/papers/w19037.ack

NBER working papers are circulated for discussion and comment purposes. They have not been peerreviewed or been subject to the review by the NBER Board of Directors that accompanies official NBER publications.

(C) 2013 by Eugenio S. A. Bobenrieth, Juan R. A. Bobenrieth, and Brian D. Wright. All rights reserved. Short sections of text, not to exceed two paragraphs, may be quoted without explicit permission provided that full credit, including $(\mathcal{C}$ notice, is given to the source. 
Bubble Troubles? Rational Storage, Mean Reversion and Runs in Commodity Prices. Eugenio S. A. Bobenrieth, Juan R. A. Bobenrieth, and Brian D. Wright

NBER Working Paper No. 19037

May 2013, Revised May 2013

JEL No. C52,Q11

\section{ABSTRACT}

High and volatile prices of major commodities have generated a wide array of analyses and policy prescriptions, including influential studies identifying price bubbles in periods of high volatility. Here we consider a model of the market for a storable commodity in which price expectations are unbounded. We derive its implications for price time series and empirical tests of price behavior. In this model commodity price is equal to marginal consumption value, and hence bubbles as defined in financial economics cannot occur. However the model generates episodes of price runs that could be characterized as "explosive" and might seem to be bubble-like. At sufficiently long holding periods, a price path can yield average returns consistent with mean reversion, even though the long run expectation of price is infinite.

Eugenio S. A. Bobenrieth

Pontificia Universidad Católica de Chile

Santiago, Chile

ebobenrieth@uc.cl

Juan R. A. Bobenrieth

Universidad del Bío-Bío

jbobenri@ubiobio.cl
Brian D. Wright

University of California, Berkeley

Agriculture and Resource Economics

bwright@berkeley.edu 


\section{Introduction}

Recent volatility of prices of major grains has revealed new interest in understanding the price behavior of storable commodities such as grains. A well-grounded model of a market for a storable staple product subject to random shocks to excess supply has been available since Gustafson (1958). Its basic logic of inter-temporal arbitrage is widely accepted, and it can generate price series that have large "spikes" and "runs" of the type that attract the concern of consumers and policy makers. However models of this type have been little used in recent analyses of commodity price fluctuations.

There are two key reasons. One is the absence of empirical support. For more than three decades the model could not be seriously tested, due to lack of both appropriate data and a satisfactory estimation procedure. When a version of the Gustafson model was eventually tested (Deaton and Laroque 1992, 1995, 1996), it was roundly rejected due to failure to replicate the high levels of serial correlation observed in commodity price data. Cafiero et al. (2011), after solving a problem of numerical accuracy in the Deaton and Laroque estimation procedure, derived estimates for several commodities consistent with the observed price correlations.

A second reason is the common impression that commodity prices can occasionally exhibit bubble-like behavior in which conditional price expectations rise without bound, and that the storage model cannot satisfactorily replicate such behavior. Recent tests have in some cases detected price "exhuberance" in observations of sporadic runs of prices of securities rising faster than the rate of interest. Some authors (for example Phillips et al. 2011) have related such price behavior to former United States Federal Reserve Bank Chairman Greenspan's remark in December 1996 regarding "irrational exhuberance" of asset prices. Researchers including Gilbert (2010) and Gutierrez (2012) have looked for similar behavior in commodity markets. Others believe that the existence of bubbles in recent grain price data is obvious only after they "crash." (Timmer 2009).

In models in the tradition of Gustafson (1958), (including Samuelson 1971, Gardner 1979, Newbery and Stiglitz 1981, and Wright and Williams 1982), the conditional expectation of price at far horizons is bounded. In their pioneering model of commodity price behavior with responsive supply, Scheinkman and Schechtman (1983, p.433) presented a model in which, if price at zero harvest is infinite, and zero harvest has positive probability, then the long run conditional expectation of price is unbounded. They inferred that in this case "the model is exactly like an exhaustible resource model. Since stocks are always held, discounted price must exceed today's price by the marginal cost of storage. This seems very unrealistic behavior for the price of a producible commodity". Since continuously increasing price is something not observed in commodity markets (in contrast to price spikes, or price runs that eventually "crash"), their decision to restrict attention to models in which stocks carried to the next period are zero so that "mean reversion" occurs when available supply is below some strictly positive level is understandable.

Several studies have identified "mean reversion" variously defined, in commodity prices, adding empirical support to the informal inference of Scheinkman and 
Schechtman (1983) that the standard model of storage must have occasional "stockouts." (i.e. periods with zero discretionary stocks).

After a brief review of the issues regarding consistency of the standard version of the model with observed time series of prices, we focus on the questions regarding the capacity of the model to replicate bubble behavior, and mean reversion. We draw on Scheinkman and Schechtman (1983) and Bobenrieth et al. (2002) to derive new implications for price behavior, and simulate an example of price realizations in which the conditional expectation of price goes to infinity. We then establish some empirical implications for sample averages of returns from time series of prices, and relate these to findings of mean reversion.

\section{The Model}

In this paper we use a stylized model of a market for a storable commodity such as a food grain, to reconsider the capacity of storage arbitrage to replicate key features of commodity price behavior identified in empirical studies. We model a competitive market for a single storable consumption commodity such as a food grain in which time is discrete and all agents have rational expectations, in which the price process has an invariant distribution similar to that of Scheinkman and Schechtman (1983) and Bobenrieth, Bobenrieth and Wright (2002). The distribution of the harvest disturbance can have an atom at its minimum value, here normalized at zero, and price at zero consumption is infinite.

Production is subject in each period to a common exogenous i.i.d. disturbance $\omega \in[0, \bar{\omega}], 0<\bar{\omega}<\infty$. The distribution of $\omega$ is of the form $\alpha L_{d}+(1-\alpha) L_{c}$, where $\alpha \in[0,1], L_{d}$ is a discrete distribution with a unique atom at 0 , and $L_{c}$ is an absolutely continuous distribution, with continuous derivative when restricted to its support $[0, \bar{\omega}]$.

Assume that there is a continuum of identical producers, a continuum of identical storers, and a continuum of identical consumers; each of the three has total measure one. There is a one-period lag between the producers' choice of effort $\lambda \geq 0$ and output of the commodity $\omega^{\prime} \lambda$, where $\omega^{\prime}$ is next period's harvest shock. Cost of effort is given by a function $g: \mathbb{R}_{+} \rightarrow \mathbb{R}_{+}$, with $g(0)=0, g^{\prime}(0)=0$, and $g^{\prime}(\lambda)>0, g^{\prime \prime}(\lambda)>$ 0 for all $\lambda>0$. Storers can hold any non-negative amount of available supply from one year to the next, and then these stocks are all available for consumption or for further storage.

We replace the key assumption of Scheinkman and Schechtman (1983) that the physical storage cost function is strictly convex and its derivative appears additively in the Euler equation with the assumption that the physical storage cost function is zero; the sole cost of storage is the cost of capital invested. Given storage $x$ and effort $\lambda$, the next period's total available supply is $z^{\prime} \equiv x+w^{\prime} \lambda$. Producers and storers are risk neutral and have a common constant discount factor $\delta \equiv 1 /(1+r)$, where $r>0$ is the discount rate.

The utility function of the representative consumer $U: \mathbb{R}_{+} \rightarrow \mathbb{R}_{+}$is continuous, once continuously differentiable, strictly increasing and strictly concave. It satisfies 
$U(0)=0, U^{\prime}(0)=\infty .{ }^{1}$ The inverse consumption demand curve, with zero income elasticity, is then $f=U^{\prime}{ }^{2}$ We assume $U$ has a finite upper bound, and thus total revenue $c f(c)$ is also bounded, and that the expectation of $f$ with respect to $L_{c}$ is finite. ${ }^{3}$ The perfectly competitive market yields the same solution as the surplus maximization problem. The Bellman equation for the surplus problem is:

$$
\begin{gathered}
\nu(z)=\max _{x, \lambda}\left\{U(z-x)-g(\lambda)+\delta E\left[\nu\left(z^{\prime}\right)\right]\right\}, \quad \text { subject to } \\
z^{\prime}=x+\omega^{\prime} \lambda, \\
x \geq 0, \quad z-x \geq 0, \quad \lambda \geq 0,
\end{gathered}
$$

where $E[$.$] denotes the expectation with respect to next period's productivity shock$ $\omega^{\prime}$.

By standard results (see for example Stokey and Lucas with Prescott, 1989), $\nu$ is continuous, strictly increasing, strictly concave, and the optimal storage and effort functions $x(z)$ and $\lambda(z)$ are single valued and continuous.

Consumption and price are given by the functions $c(z) \equiv z-x(z), p(z) \equiv$ $f(z-x(z))$.

The storage and effort functions $x$ and $\lambda$ satisfy the Euler conditions:

$$
\begin{aligned}
& f(z-x(z)) \geq \delta E\left[\nu^{\prime}\left(x(z)+\omega^{\prime} \lambda(z)\right)\right], \quad \text { with equality if } x(z)>0 \\
& g^{\prime}(\lambda(z)) \geq \delta E\left[\omega^{\prime} \nu^{\prime}\left(x(z)+\omega^{\prime} \lambda(z)\right)\right], \quad \text { with equality if } \lambda(z)>0
\end{aligned}
$$

and the envelope condition $\nu^{\prime}(z)=f(z-x(z))$.

Given initial available supply $z>0$, if the probability of zero productivity shock, $\alpha$, is strictly positive, condition (1) implies that $z^{\prime}>0$ and $x\left(z^{\prime}\right)>0$, and this arbitrage condition holds with equality in the current period and for the indefinite future. When positive, storage $x(z)$ is strictly increasing with $z$, and effort $\lambda(z)$ is decreasing with $z$. Note that $p(0)=f(0)=\infty$.

Define available supply at time $t$ as $z_{t}$. Given arbitrary fixed $z_{0}>0$, the function that yields the supremum of the support of $z_{t+1}$ is $\hat{z}\left(z_{t}\right) \equiv x\left(z_{t}\right)+\lambda\left(z_{t}\right) \bar{\omega}$. From the fact that there exists a unique fixed point $z^{*}$ of $\hat{z}$ such that $\hat{z}(z)<z$ for all

\footnotetext{
${ }^{1}$ Unbounded marginal utility implies no substitution in the margin, an assumption which is more plausible for aggregate rather than for individual food commodities.

${ }^{2}$ As discussed in footnote 1 of Scheinkman and Schechtman 1983, specification of a quasilinear utility function is one way to incorporate income, in the setting of general equilibrium models that generate the same set of equilibria as this partial equilibrium specification.

${ }^{3}$ This guarantees that for a model with harvest disturbances with distribution $L_{c}$ there is a finite threshold price above which discretionary stocks are zero.
} 
$z>z^{*}$, we conclude that $z_{t} \leq \bar{z} \equiv \max \left\{z_{0}, \max \left\{\hat{z}(z): 0 \leq z \leq z^{*}\right\}\right\}$, for all $t \geq 0$. Then a suitable state space is $S \equiv[0, \bar{z}]$. Storage takes values in the set $[0, \bar{x}]$, where $\bar{x} \equiv x(\bar{z})$.

\section{Empirical Relevance Revisited}

\subsection{Failure to match observed high price correlations}

Deaton and Laroque $(1992,1995,1996)$ presented empirical tests of the Gustafson model (1958) using first simulations of the model, and then econometric estimates based on the short available annual time series of prices of a number of commodities. Deaton (forthcoming, 2012) summarizes their overall conclusion:

"We have a long-established theory - whose insights are deep enough that some part of them must be correct - which is wildly at odds with the evidence, and where it is far from obvious what is wrong [...]."

Cafiero et al. (2011) show, first, that a version of the Gustafson model with lower consumption demand elasticity, can generate the high levels of serial correlation observed in commodity prices. Second, they show that application of Deaton and Laroque's $(1995,1996)$ econometric approach, modified to improve its numerical accuracy, using the same data set, yields empirical results that are consistent with observed levels of price variation and autocorrelation for seven major commodities. In a subsequent paper, Cafiero et al. (2012) derive maximum likelihood estimates that impose no more assumptions than the previous pseudo-maximum likelihood estimates, for the global sugar market, and obtain even better results.

Thus we are now in a position to consider the relevance of the Gustafson model for interpreting and testing recent claims regarding the behavior of commodity prices. In particular, we address in this paper claims that grain markets display "mean reversion," or that they have recently been disrupted by "bubbles" (Gilbert 2010, Piesse and Thirtle, 2009,Timmer 2009, 2010, Gutierrez 2012), or by "exhuberant" behavior (Phillips et al. 2011), and by the popular notion that such claims can be resolved, at least in principle, from observed price behavior.

The model tested by Deaton and Laroque $(1992,1995,1996)$ and Cafiero et al. $(2011,2012)$ assumes linear demand, with stocks that go to zero at a finite price. To address questions about mean reversion, speculative runs and related phenomena, we have adopted a demand specification that, if $\alpha>0$, does not impose mean reversion at high prices, and allows for unbounded price expectations. ${ }^{4}$ Thus our model is capable of producing behavior that includes conditional expectations of prices that go to infinity as the horizon recedes, as observed by Scheinkman and Schechtman (1983). But, is this extension of the model of any empirical relevance to actual price behavior in commodities such as grains?

${ }^{4}$ As for the linear case, questions have been raised about the realism of the behavior of prices in that model. 


\subsection{Behavior of the model with unbounded conditional price expectations}

Scheinkman and Schechtman (1983) stated that price behavior when $\alpha>0$ and price at zero consumption is infinite is very unrealistic for a producible commodity, since it is "exactly like that of a natural resource model in which discounted price rises by the marginal cost of storage." Thus we start by considering a case of the model which is in fact a natural resource model.

\subsubsection{The deterministic finite natural resource model}

If $\alpha=1$ then our model, which has no storage cost, is the deterministic Hotelling model of consumption of a finite resource with unbounded price. Standard results are that price rises monotonically at the rate of interest, so that discounted future prices equal the current price. Such price behavior is indeed inconsistent with actual stochastic evolution of prices for commodities such as food grains. Does this price behavior generalize to the case in which $0<\alpha<1$ ?

\subsubsection{The stochastic model with unbounded price expectations}

Intertemporal storage arbitrage implies that, in the model with $0<\alpha<1$, $\left\{\delta^{t} p_{m+t}\right\}_{t \geq 0}$ is a martingale and $\left\{E_{m}\left[\delta^{t} p_{m+t}\right]: t \geq 0\right\}=p_{m}$, where $E_{m}[$.$] denotes$ the expectation conditional on the price $p_{m}$ at time $m$. Indeed the conditional expectation of price behaves exactly as the price in the deterministic natural resources model discussed above. But in this stochastic model the price path does not follow its expectation, contrary to the inference of Scheinkman and Schechtman (1983). Nor does the statement of Bessembinder et al. (1995 p. 362) that the path of conditional expectations at different horizons "describes several points on the path that investors expect the spot price will take" hold for this model. To the contrary, as the horizon recedes, the path of realized prices eventually drifts down and away from the rising profile of conditional expectations, any fraction of which becomes an upper bound on that realized path.

The sequence of probability measures of prices conditional on any initial price $p_{m}$ converges to a unique invariant measure, uniformly in $p_{m}$, and consequently the sequence of discounted prices converges in probability to zero, uniformly in $p_{m} .{ }^{5}$

More precisely:

THEOREM 1: Let $\alpha<1$. Given $\beta>0$ and $\varepsilon>0$, there exists $T \in \mathbb{N}$ such that for any price realization $p_{m}$,

\footnotetext{
${ }^{5}$ If $0 \leq \alpha<1$, the sequence of probability measures of $z_{t},\left\{\gamma_{t}\right\}_{t=0}^{\infty}$, converges in the total variation norm to a unique invariant probability $\gamma_{*}$, regardless of the value of $z_{0}$. The idea of the proof for the case $0 \leq \alpha<1$ can be found in Bobenrieth, Bobenrieth and Wright (2002). It follows immediately that the sequence of probability measures of prices $\left\{\gamma_{t} c^{-1} f^{-1}\right\}_{t=0}^{\infty}$ converges in the total variation norm to the unique invariant probability measure $\gamma_{*} c^{-1} f^{-1}$. Note that $\operatorname{Prob}\left[p_{t} \geq y\right]=\left(\gamma_{t} c^{-1} f^{-1}\right)([y, \infty])$, where $p_{t}=f\left(c\left(z_{t}\right)\right)$ is the price at time $t . H_{t}(y) \equiv \operatorname{Prob}\left[p_{t} \geq y\right]$ converges uniformly to a unique invariant upper c.d.f. $H_{*}$, with $\lim _{p \rightarrow \infty} H_{*}(p)=0$. If $0 \leq \alpha<1$, then the support of the invariant distribution of prices is an interval $[\underline{p}, \infty]$ with $0<\underline{p}<\infty$.
} 


$$
\operatorname{Prob}\left[\delta^{t} p_{m+t}<\beta \mid p_{m}\right] \geq 1-\varepsilon, \quad \forall t \geq T \text {. }
$$

Theorem 1 implies that for any sample size $N \in \mathbb{N}$, given any finite sequence of realized initial prices $\left\{p_{m}, p_{m+1}, \cdots, p_{m+N-1}\right\}$, we have the following bound on the joint probability of the gross discounted relative price changes from each initial price in the sample, beyond a finite $T^{\prime}$, where $T^{\prime}$ is independent of the finite sequence of initial price realizations:

$$
\operatorname{Prob}\left[\frac{\delta^{t} p_{m+t}}{p_{m}}<\beta, \frac{\delta^{t} p_{m+1+t}}{p_{m+1}}<\beta, \cdots, \frac{\delta^{t} p_{m+N-1+t}}{p_{m+N-1}}<\beta \quad \mid p_{m+N-1}\right] \geq 1-\varepsilon,
$$

for all $t \geq T^{\prime}{ }^{6}$

The existence of a unique invariant distribution which is a global attractor implies for this price process that, with probability one, the sequence of price realizations is dense on the support $[p, \infty]$ of the invariant distribution. The infinite sequence of price realizations visits every neighborhood of every price in the support, no matter how high, infinitely often, almost surely. Given this fact, the following proposition regarding discounted prices might not be surprising:

PROPOSITION 1: Let $\alpha<1$. For any given price realization $p_{m}$, for arbitrary positive real number $D$, there exists a horizon $d \in \mathbb{N}$, such that:

$$
\operatorname{Prob}\left[\delta^{t} p_{m+t}>D \mid p_{m}\right]>0, \quad \forall t \geq d
$$

For the case $0<\alpha<1$, the maximum of the support of the conditional distribution of discounted price goes to infinity as the horizon increases, in contrast to the case for the standard Gustafson model with bounded price, where the maximum goes to zero. To prove Proposition 1, we need Proposition 2, which might seem counter-intuitive given Proposition 1.

For the discussion that follows, given a price realization $p_{m}$, let $E_{m}[$.$] denote the$ expectation conditional on $p_{m}$.

PROPOSITION 2: Let $\alpha<1$. Given any price realization $p_{m}$, the sequence of discounted prices, $\left\{\delta^{t} p_{m+t}\right\}_{t \geq 0}$, goes to zero, almost surely (as $t \rightarrow \infty$ ).

Proof of Proposition 2: The Euler condition for storage arbitrage (1) implies that, if $\alpha>0,\left\{\delta^{t} p_{m+t}\right\}_{t \geq 0}$ is a martingale and $\sup \left\{E_{m}\left[\delta^{t} p_{m+t}\right]: t \geq 0\right\}=$ $p_{m}<\infty$. In the case $\alpha=0,\left\{\delta^{t} p_{m+t}\right\}_{t \geq 0}$ is a supermartingale and $\sup \left\{E_{m}\left[\delta^{t} p_{m+t}\right]\right.$ :

${ }^{6}$ In the proof, presented in the Appendix, we use the facts that the Markov operator is stable and quasicompact, and that given any initial price, any neighborhood of infinity, and any integer $k$, the price process visits that neighborhood in a time that is some multiple of $k$, with positive probability. 
$t \geq 0\}=p^{*}<\infty$. In both cases, by the Martingale Convergence Theorem (due to Doob) we conclude that $\delta^{t} p_{m+t} \rightarrow Y$ a.s. (as $t \rightarrow \infty$ ), where $Y$ is a real random variable. By Theorem $1, \delta^{t} p_{m+t} \rightarrow 0$ in probability (as $t \rightarrow \infty$ ), and hence $Y=0$ almost surely.

Q.E.D.

Proof of Proposition 1: For the nontrivial case $0<\alpha<1$, we prove the result by contradiction. If not, there exist a price realization $p_{m}$, a real number $D>0$ and a sequence of natural numbers $\left\{t_{k}\right\}_{k \in \mathbb{N}} \uparrow \infty$ with $\operatorname{Prob}\left[\delta^{t_{k}} p_{m+t_{k}}>\right.$ $\left.D \mid p_{m}\right]=0$, for all $t_{k}$. Therefore $\delta^{t_{k}} p_{m+t_{k}} \leq D$ a.s., for all $t_{k}$. Then the Lebesgue dominated convergence theorem and the fact that $\lim _{t_{k} \rightarrow \infty} \delta^{t_{k}} p_{m+t_{k}}=0$ a.s. imply that $\lim _{t_{k} \rightarrow \infty} E_{m}\left[\delta^{t_{k}} p_{m+t_{k}}\right]=0$, a contradiction to $E_{m}\left[\delta^{t_{k}} p_{m+t_{k}}\right]=p_{m}>0$, for all $t_{k}$. Q.E.D.

If $0<\alpha<1$, we have that $E_{m}\left[\delta^{t} p_{m+t}\right]=p_{m}, \forall t \geq 0$. Nevertheless, Proposition 2 states $\left\{\delta^{t} p_{m+t}\right\}_{t \geq 0}$, converges to zero almost surely, implying that $\left\{E_{m}\left[\delta^{t} p_{m+t}\right]\right\}_{t \geq 0}$ does not converge to the expectation of the almost sure limit of $\left\{\delta^{t} p_{m+t}\right\}_{t \geq 0}$. As a consequence, the sequence of discounted prices is not uniformly integrable.

Proposition 2 is easy to understand in a model with $\alpha=0$, but if $0<\alpha<1$, how can the discounted price be going to zero, almost surely, if there is positive probability that discounted price exceeds $D$ at any sufficiently far horizon? The explanation hinges on the distinction drawn above between a profile of expectations conditional on a price realization and the path of realizations. By Proposition 2, with probability one, for any given path of discounted price realizations there is a time beyond which that path is permanently below $D$. But by Proposition 1, there is no finite horizon beyond which all paths possible from date $m$ are below $D$. In fact, at any finite horizon, with positive probability price rises at a rate greater than the discount rate $r$, continuously within that horizon. Although any path of discounted price realizations eventually remains permanently below $D$, before it does so, it can exceed any given arbitrary high finite bound. It is recognition of such a possibility that keeps $E_{m}\left[\delta^{t} p_{m+t}\right]$ equal to $p_{m}$ as the horizon, and the probability that the discounted price will be below $D$ at that horizon, both increase.

Proposition 2 implies that, given a price realization $p_{m}$, the sample mean and sample variance of a discounted price sequence go to zero almost surely, that is: 


$$
\begin{gathered}
N^{-1} \sum_{t=0}^{N-1} \delta^{t} p_{m+t} \rightarrow 0 \quad \text { a.s. } \quad(\text { as } N \rightarrow \infty), \quad \text { and } \\
N^{-1} \sum_{t=0}^{N-1}\left[\delta^{t} p_{m+t}-N^{-1} \sum_{j=0}^{N-1} \delta^{j} p_{m+j}\right]^{2} \rightarrow 0 \quad \text { a.s. } \quad(\text { as } \quad N \rightarrow \infty) .
\end{gathered}
$$

Thus the estimators are consistent with respect to the first two moments of the limiting distribution of discounted price. For the case $0<\alpha<1$, the sample average of discounted price realizations starting at any price realization $p_{m}$, is eventually permanently below any arbitrary positive fraction of the profile of expectations, conditional on $p_{m}$, of discounted price. Nevertheless the variance of the distribution of discounted price, conditional on $p_{m}$, goes to infinity as $t \rightarrow \infty$.

The behavior of the price path is related to the profile of conditional expectations at time $m$ by the following theorem:

THEOREM 2: Let $0<\alpha<1$. Given any price realization $p_{m}$, with probability one, for any $1 \leq l<\infty$, there exists a finite time $\tau(l)$, which depends on the sequence of price realizations, such that:

$$
\frac{E_{m}\left[p_{m+t}\right]}{l}>p_{m+t}, \quad \forall t \geq \tau(l)
$$

implying that

$$
p_{m+t}=o\left(E_{m}\left[p_{m+t}\right]\right), \quad \text { a.s. }
$$

Proof of Theorem 2: By Proposition 2, $\delta^{t} p_{m+t} \rightarrow 0$ (as $t \rightarrow \infty$ ), with probability one. Therefore, given any $l, 1 \leq l<\infty$, there exists a time $\tau(l)$ that satisfies $\delta^{t} p_{m+t} \cdot l<p_{m}=\delta^{t} E_{m}\left[p_{m+t}\right], \quad \forall t \geq \tau(l)$.

Q.E.D.

Theorem 2 defines a sequence of upper bounds on the path of price realizations. Note that the profile of conditional expectations $E_{m}\left[p_{m+t}\right]$ is itself an upper bound beyond some date $\tau(1)$. Any given fraction of the profile of expectations conditional on initial price is an upper bound on any price realized beyond some fixed horizon, with probability one. 


\section{Price Behavior in this Model: Do We See Bub- bles?}

The behavior of price expectations and realizations in the model is illustrated in the example in Figure 1. At time 0 profile of conditional expectations, $E_{0}\left[p_{t}\right]$, rises to infinity at the discount rate. A possible sequence of price realizations is illustrated as a grey curve beginning at $p_{0} \cdot{ }^{7}$ After period 23, all the realizations of price lie below $E_{0}\left[p_{t}\right]$. The curve $E_{0}\left[p_{t}\right] / 2$ shows another bound at half the price expectations is effective beginning at date 39. It is obvious that further bounds generated by successively higher values of $l$ would imply that the long-run rate of increase of realized price is strictly lower than the discount rate, $4 \%$, even though the storage arbitrage condition (1) holds, with equality, each period, and that price runs of any finite length, understood as sequences of prices rising faster than the interest rate, recur infinitely often along the path of realizations, almost surely. Figure 2 shows the logarithms of the same price series, dramatizing the runs of price increases greater than the rate of interest.

These figures show that runs of prices rising for several years at a rate greater than the rate of interest before crashing, denoted "explosive" by Phillips et al. (2011), and fulfilling the empirical ex post criterion for identification of bubbles in grain prices enunciated by Timmer $(2009,2010)$, are consistent with our equilibrium model with rational expectations. In this model, they do not signify the disruptive effects of irrational speculation, but rather the dampening effect of storage that prevents sharper price jumps, but with declining effectiveness if low harvests persist.

Discussions of volatile grain price behavior often raise the issue of price bubbles, frequently without defining the term. Brunnermeier (2008) includes a key feature of most definitions of finance economists when he states that "Bubbles refer to asset prices that exceed an asset's fundamental value because current owners believe they can resell the asset at an even higher price." Are price runs characteristic of price behavior in our model, as illustrated in Figures 1 and 2, consistent with this definition? In our model we have assumed no convenience yield, and the law of one price holds. In that setting, when storage is positive, the value of a commodity such as food grain equals its value in consumption, as indicated in the envelope condition after equations (1) and (2) above. Storage is a one period investment, so its "fundamental" is the market price, which derives its marginal value from its value in consumption. Thus in our model bubbles consistent with Brunnermeier's definition cannot occur. In this model, they do not signify the disruptive effects of rational or irrational speculation, but rather the dampening effect of storage that prevents sharper price jumps, but with declining effectiveness, during episodes of repeated low harvests.

${ }^{7}$ Bobenrieth, Bobenrieth and Wright (2008) offers a foundation for a strategy for numerical solution of marginal values in cases where they are unbounded. 


\section{Some Empirical Implications}

Implications of the model for the empirical behavior of sample averages of returns on the stocks, held over specific intervals, are summarized in the following theorem:

THEOREM 3: Let $0<\alpha<1$. With probability one, for any given path of price realizations $\left\{p_{t}\right\}_{t \geq 0}$, for any $n \in \mathbb{N}$ and for any $\beta>0$, there exist $J=$ $J\left(\left\{p_{t}\right\}_{t \geq 0}, n, \beta\right) \in \mathbb{N}, \quad k=k\left(\left\{p_{t}\right\}_{t \geq 0}, J, \beta\right) \in \mathbb{N}, \quad k>n$, and $K=K\left(\left\{p_{t}\right\}_{t \geq 0}, k, \beta\right) \in$ $\mathbb{N}, K>J$, such that:

(i) $J^{-1} \sum_{t=0}^{J-1}\left[\frac{\delta^{n} p_{t+n}-p_{t}}{p_{t}}\right] \in(-\beta, \beta)$,

(ii) $J^{-1} \sum_{t=0}^{J-1}\left[\frac{\delta^{k} p_{t+k}-p_{t}}{p_{t}}\right] \in(-1,-1+\beta), \quad$ and

(iii) $K^{-1} \sum_{t=0}^{K-1}\left[\frac{\delta^{k} p_{t+k}-p_{t}}{p_{t}}\right] \in(-\beta, \beta)$.

Proof of Theorem 3: For $j \in \mathbb{N}$ and for $t \in \mathbb{N} \cup\{0\}$, let $Y_{t+j} \equiv \frac{\delta^{j} p_{t+j}-p_{t}}{p_{t}}$. The arbitrage equation for storage (1) implies that there exists $\bar{p} \geq p\left(x^{j}\left(z_{t}\right)\right), \quad \bar{p}$ depends on $z_{t}$, such that $\delta^{j} \alpha_{1}^{j} \bar{p}=p_{t}$, where $\alpha_{1}$ is the size of the atom at zero of the distribution of $\omega$, and $x^{j} \equiv x \circ x \circ \cdots \circ x$ ( $j$ times). Therefore,

$$
-1 \leq Y_{t+j} \leq \delta^{j} \frac{\bar{p}}{p_{t}}=\frac{1}{\alpha_{1}^{j}}
$$

The arbitrage equation (1) also implies $E_{t}\left[Y_{t+j}\right]=0$. It follows that the sequence $\left\{X_{t}\right\}_{t \geq 0}$, where $X_{t} \equiv Y_{t+j}$, is uniformly bounded, and $\sum_{i=1}^{\infty} \sup _{t}\left|\operatorname{Cov}\left(X_{t}, X_{t-i}\right)\right|<\infty$. A strong law of large numbers (see Davidson 1994, p.297) implies that

$$
\lim _{N \rightarrow \infty} N^{-1} \sum_{t=0}^{N-1}\left[\frac{\delta^{j} p_{t+j}-p_{t}}{p_{t}}\right]=0, \quad \text { a.s. }
$$

Evaluating (2) for $j=n$ we conclude that there exists $J \in \mathbb{N}$ such that $(i)$ holds. For this $J$, by Proposition 2,

$$
\lim _{k \rightarrow \infty} J^{-1} \sum_{t=0}^{J-1}\left[\frac{\delta^{k} p_{t+k}-p_{t}}{p_{t}}\right]=-1, \quad \text { a.s. }
$$


establishing $(i i)$ for large enough $k$. Finally, evaluating (2) for $j=k$ we obtain $K, K>J$, satisfying $(i i i)$.

Q.E.D.

Expression $(i)$ of Theorem 3 shows that the average excess rate of return on stocks held over $n$ periods is greater than a given, arbitrary $-\beta$, for a sufficiently large sample size $J$, as implied by a strong law of large numbers. ${ }^{8}$ Expression (ii) states that, with the same sample of initial holding dates, if we increase the holding interval sufficiently, to $k$ periods, (and increase the sample size by $k-n$ periods to accommodate the extended lead), the average gross discounted return is within an arbitrary $\beta$ of a total loss. At this sample size, the sample average $(i i)$ could be considered a downward-biased estimator of the expected $k$-period rate of increase in price, which in this model is constant. Thus for any sample of prices of any given length, one can find a sufficiently far horizon such that the estimated average return can be taken to imply "mean reversion," as defined for example in Bessembinder et al. (1995) even if the behavior of prices does not exhibit mean reversion, as in the stationary model considered here. Expression (iii) reflects the fact that the sample average for the longer holding period approaches the conditional expectation for that horizon, when the sample size is sufficiently increased.

Comparison of results $(i)$ through $($ iii $)$ has another interpretation, more relevant for estimation of the long-run return on storage from any given time zero. As the horizon is increased, the discounted present value of price realizations conditional on any price $p_{t}$ in the sample of size $J$ in $(i)$ eventually converges, along the path of realizations, to a neighborhood of zero in finite time, as stated in Proposition 2. From this point of view, comparison of $(i i)$ with $(i)$ reflects the convergence of the gross discounted value to its almost sure limit of a one hundred percent loss over the holding period, as the latter goes to infinity. But (iii) shows an increase in the average excess rate of return back to an arbitrary neighborhood of the conditional expectation of zero when sufficient observations are added to include some that have high rates of price increase through the fixed horizon. Note that (iii) does not imply that an initial investment at time zero can be restored to profitability if held for a sufficiently long time.

\section{Conclusions}

The remarkable work of Gustafson (1958) introduced a market model that numerically derives the storage demand given consumer demand, yield distribution, cost of storage and interest rate, assuming maximization of expected profits. The standard model shows why price distributions tend to be skewed, and why do not closely reflect production shocks. Recent empirical results confirm that it can, contrary to previous claims, also match the high price correlations seen in annual prices of major commodities. However the model as presented by Gustafson cannot address

${ }^{8} \mathrm{~A}$ similar result is confirmed (on a very different time scale) for daily returns for wheats on the Kansas City and Minneapolis grain exchanges in Bobenrieth (1996). 
the behavior of prices if their profile of conditional expectations are unbounded, as in some models of speculative behavior.

Here we consider an extension of the Gustafson model, introduced in Scheinkman and Schechtman (1983) and addressed by Bobenrieth et al. (2002), in which price expectations are unbounded, and derived its implications for price time series and empirical tests of price behavior. We present versions of the model that exhibit price behavior that could be characterized as "explosive" or "exhuberant" with episodes of price runs that might be identified as "bubbles." In this model, conditional price expectations go to infinity as the horizon recedes, consistent with stationary behavior. This behavior is indistinguishable from that produced by a version of the standard model with bounded conditional price expectations, so a test to establish that price is unbounded is infeasible.

The stationary price process that we have examined reveals the importance of distinguishing any given profile of conditional price expectations from the path of price realizations. The rate of increase of any profile of conditional price expectations in our model is constant at the discount rate, while beyond some future period the path of realized prices lies permanently below the profile of expectations conditional on the current price. Returns on storage are returns consistent with "mean reversion" at sufficiently long holding periods, even though the long run expectation of price is infinite. 


\section{REFERENCES}

Bessembinder, H., J. F. Coughenour, P. J. Seguin and M. Smaller (1995): "Mean Reversion in Equilibrium Asset Prices," The Journal of Finance, 50(1), 361-375.

Bobenrieth, E. S. A. (1996): "Commodity Prices Under Time-Heterogeneous Shocks Density," Ph.D. Thesis.University of California at Berkeley.

Bobenrieth, E. S. A., J. R. A. Bobenrieth, And B. D. Wright (2002): "A Commodity Price Process With a Unique Continuous Invariant Distribution Having Infinite Mean," Econometrica, 70, 1213-1219.

(2008): "A Foundation for the Solution of Consumption-Saving Behavior with a Borrowing Constraint and Unbounded Marginal Utility," Journal of Economic Dynamics $\&$ Control, 32, 695-708.

BrunnermeIER, M. K. (2008): “Bubbles.” New Palgrave Dictionary of Economics, Second Edition.

Cafiero, C., E. S. A. Bobenrieth, J. R. A. Bobenrieth, and B. D. WRIGHT (2011): "The Empirical Relevance of the Competitive Storage Model," Journal of Econometrics, 162, 44-54.

(2012): "Can Simple Storage Arbitrage Explain Commodity Price Dynamics? Evidence from Sugar Prices," Working Paper FAO, Pontificia Universidad Católica de Chile, Universidad del Bío-Bío, and University of California at Berkeley.

DAVIDSON, J. (1994): Stochastic Limit Theory. Oxford University Press.

DEATON, A. (forthcoming, 2012): "Puzzles and paradoxes: a life in applied economics." in Michael Szenberg and Lall Ramrattan, eds., Eminent Economists II, Cambridge University Press.

Deaton, A., And G. LARoque (1992): "On the Behaviour of Commodity Prices," Review of Economic Studies, 59, 1-23.

(1995): "Estimating a Nonlinear Rational Expectations Commodity Price Model with Unobservable State Variables," Journal of Applied Econometrics, 10, S9-S40.

(1996): "Competitive Storage and Commodity Price Dynamics," Journal of Political Economy, 104, 896-923. 
FutiA, C. A. (1982): "Invariant Distributions and Limiting Behavior of Markovian Economic Models," Econometrica, 50, 377-408.

GARDNER, B. L. (1979): Optimal Stockpiling of Grain. Lexington Books, Lexington, Mass.

GilberT, C. L. (2010): "Speculative Influences on Commodity Futures Prices 20062008," UNCTAD Discussion paper.

Gustafson, R. L. (1958): "Carryover Levels for Grains," USDA Technical Bulletin 1178, Washington, DC: United States Department of Agriculture.

Gutierrez, L. (2012): "Speculative Bubbles in Agricultural Commodity Markets," European Review of Agricultural Economics, doi: 10.1093/erae/jbs017

NewBery, D. M., AND J. E. STIGLITZ (1981): The Theory of Commodity Price Stabilization: A Study in the Economics of Risk. New York : Oxford university Press.

Phillips, P. C. B., Y. WU, AND J. YU (2011): "Explosive Behavior in the 1990s NASDAQ: When Did Exuberance Escalate Asset Values?," International Economic Review, 162, 44-54.

Piesse, J., AND C. ThiRTle (2009): "Three Bubbles and a Panic: An Explanatory Review of Recent Food Commodity Price Events," Food Policy, 34 (2), 119-129.

SAmuelson, P. (1971): "Stochastic Speculative Price," Proceedings of the National Academy of Sciences, 68, 335-337.

SCHEINKMAN, J. A., AND J. SCHECHTMAN (1983): "A Simple Competitive Model with Production and Storage," Review of Economic Studies, 50, 427-441.

Stokey, N. L., R. E. LucAs, JR., With E. C. PRescotT (1989): Recursive Methods in Economic Dynamics. Cambridge, MA: Harvard University Press.

Timmer, C. P. (2009): "Did Speculation affect World Rice Prices ?," Working Paper prepared for the FAO Conference on "Rice Policies in Asia" held in Chiang Mai, Thailand from February 9 - 12, 2009.

Timmer, C. P. (2010): "Reflections on Food Crises Past," Food Policy, 35(1), 1 11.

Wright, B. D., AND J. C. Williams (1982): "The Economic Role of Commodity Storage," The Economic Journal, 92, 596-614.

YosidA, K., AND S. KAKUTANI (1941): "Operator-Theoretical Treatment of Markoff's Process and Mean Ergodic Theorem," Annals of Mathematics, 42, 188-228. 
APPENDIX. PROOF OF THEOREM 1: Consider the probability of the complement,

$$
\operatorname{Prob}\left[\delta^{t} p_{m+t} \geq \beta \mid p_{m}\right]=\operatorname{Prob}\left[p_{m+t} \geq \frac{\beta}{\delta^{t}} \mid p_{m}\right]=\mu_{t}\left(\left[\frac{\beta}{\delta^{t}}, \infty\right]\right)
$$

where $\mu_{t}$ is the probability measure of the price at time $m+t$, conditional on $p_{m}$. Furthermore,

$$
\mu_{t}\left(\left[\frac{\beta}{\delta^{t}}, \infty\right]\right) \leq\left|\mu_{t}-\mu_{*}\right|+\mu_{*}\left(\left[\frac{\beta}{\delta^{t}}, \infty\right]\right)
$$

where $\mu_{*}$ is the invariant probability measure of the price process and $|\cdot|$ denotes the total variation norm.

The transition probability of the price process satisfies, with respect to the point $\infty$, what is called in Futia a Generalized Uniqueness Criterion (Futia, 1982, p.390). In addition, the corresponding Markov operator $L$ is stable and quasicompact (Theorems 4.6 and 4.10 in Futia, 1982, p.394 and p. 397). Using Theorem 3.6 in Futia (1982, p.390), and Theorem 4 in Yosida and Kakutani (1941, p.200), we obtain the following conclusion: independent of $p_{m}$, there exist constants $M>0, \eta>0$, such that :

$$
\left\|\left(L^{*}\right)^{t}-L_{1}^{*}\right\| \leq \frac{M}{(1+\eta)^{t}} \quad \forall t \in \mathbb{N},
$$

where $L^{*}$ is the adjoint of the Markov operator $L, L_{1}^{*}$ is a continuous linear operator, the image of which consists precisely of the fixed points of $L^{*}$, and $\|\cdot\|$ is the operator norm. Therefore, if $\delta_{p_{m}}$ denotes the unit point mass at $p_{m}$, then :

$$
\left|\mu_{t}-\mu_{*}\right|=\left|\left(L^{*}\right)^{t}\left(\delta_{p_{m}}\right)-L_{1}^{*}\left(\delta_{p_{m}}\right)\right| \leq \|\left(L^{*}\right)^{t}-L_{1}^{*}|| \leq \frac{M}{(1+\eta)^{t}} \forall t \in \mathbb{N} .
$$

Finally, since $\mu_{*}$ has no atom at infinity, we have that $\lim _{t \rightarrow \infty} \mu_{*}\left(\left[\frac{\beta}{\delta^{t}}, \infty\right]\right)=0$.

Q.E.D. 


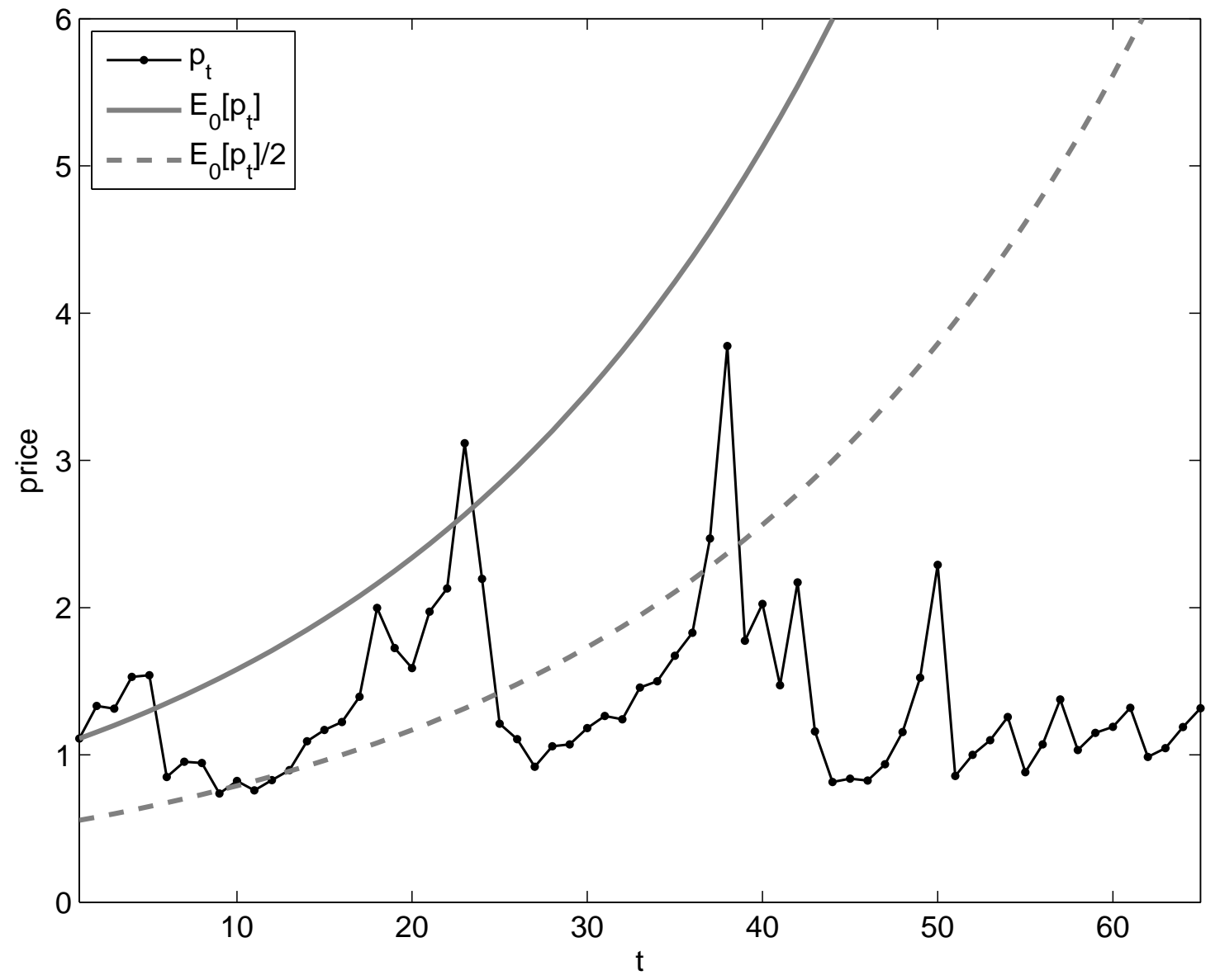

Figure 1. 


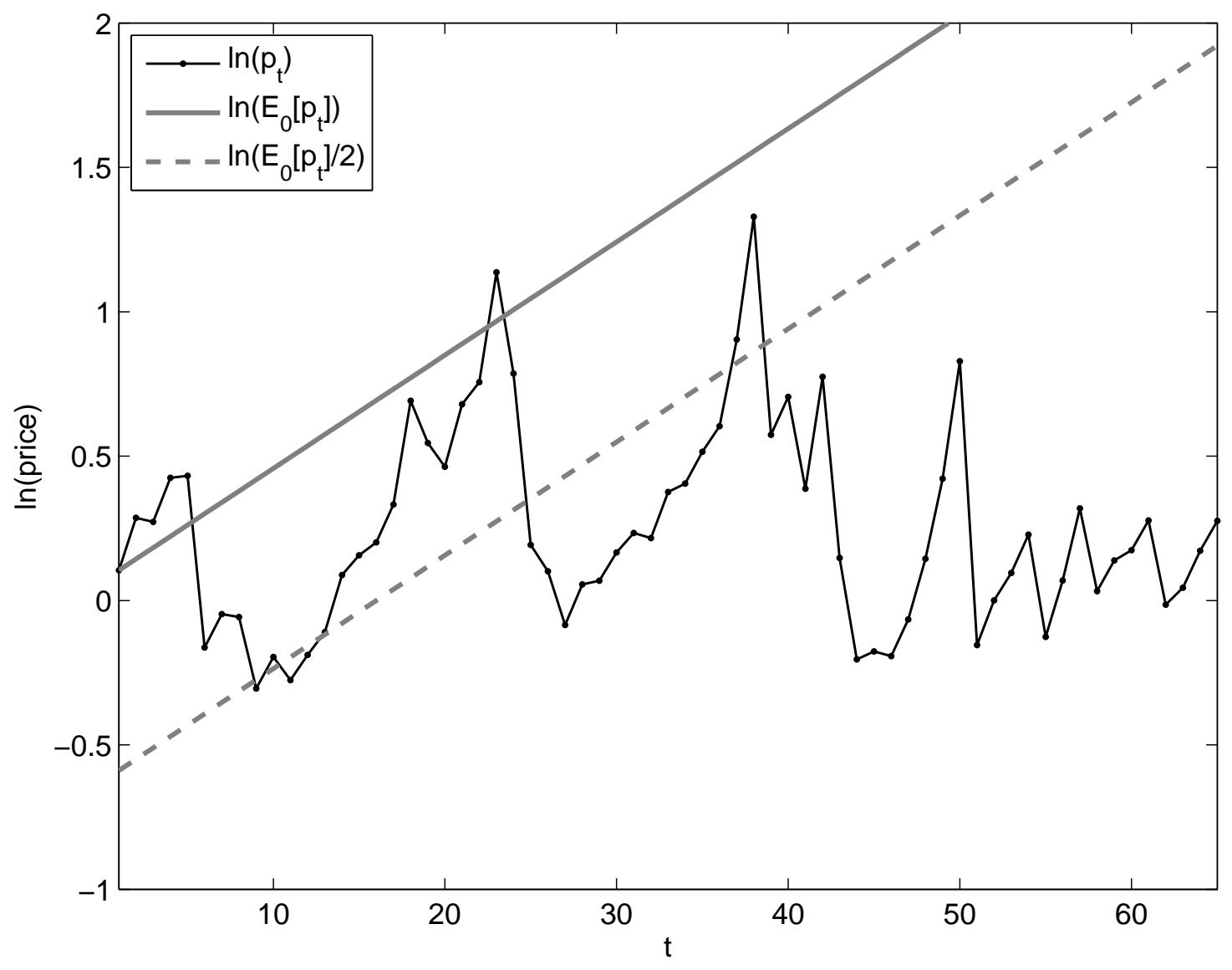

Figure 2. 